\title{
Research on the Drawing Method of Energy Sankey Diagram Based on Java
}

\author{
Chunyu DENG*, Hongmin LI**, Yupeng SHAO* \\ * Beijing Guo Dian Tong Corporation, China \\ dengchunyu@sgepri.sgcc.com.cn, lihongmin@sgepri.sgcc.com.cn, shaoyupeng@sgepri.sgcc.com.cn
}

\begin{abstract}
Energy consumption and efficiency is an important basis of a country's energy strategy formulation. Sankey Diagram has been more and more widely applied in energy consumption research as an intuitive tool to demonstrate historical energy consumption. However, the complexity of the drawing nice Sankey diagram becomes the bottleneck limits efficiency of energy researchers. As a result, an urgent demand has been derived for a graphics software that is able to automatically generate Energy Sankey Diagram based on Energy Balance Table. This paper studies drawing Energy Sankey Diagram based on Java Graphics2D, and introduces a multi arc seamless bonding curve drawing method. Experiments show that the method can not only flexibly generate flow curves, but also reduce the crosses between curves and thus makes diagrams clear and precise.
\end{abstract}

Keywords — Energy Sankey Diagram, Graphics2D, Multi Arc Seamless Bonding

\section{INTRODUCTION}

Energy consumption and efficiency is an important basis of a country's energy strategy formulation. As an intuitive tool to demonstrate historical energy consumption, Sankey Diagram has been more and more widely applied in energy consumption research. However, the complexity of the drawing nice Sankey diagram becomes the bottleneck limits efficiency of energy researchers. As a result, an urgent demand has been derived for graphics software that is able to automatically generate Energy Sankey Diagram based on Energy Balance Table. This paper studies drawing Energy Sankey Diagram based on Java Graphics2D, and introduces a multi arc seamless bonding curve drawing method.

\section{Current Situation}

At present, there are two kinds of tools for drawing Sankey diagram: e! Sankey and sankey.js. Between them, e!Sankey provides a common element representation for energy, and flows can be freely adjusted. However, it's still an inevitable process for users to manually determining the rectangular frame size, line thickness, as well as line positions. In comparison, sankey.js developed by the D3.js can automatically generate images. Yet, functional extension seems beyond the grasp of sankey.js. In addition, JavaScript supports data formats like JSON. Thus each change of data requests revision of JSON strings, which is obviously extra time consumption.
Java Graphics2D graphical programming can solve these problems. By invoking Excel spread sheets from the background, the program automatically calculates the size of each element, and then generates a Sankey diagram of the year. Thus, when data changes, users only need to modify their excel spread-sheets, and restart the program.

\section{RELATED WORK}

\section{A. Scheme Selection}

When it comes to drawing curves with java Graphics2D, there are three options:

(1) Using a quadratic curve function, namely QuadCurve2d;

(2) Using a cubic curve function, namely CubicCurve2d;

(3) Self-constructed curve.

The first scheme can generate unidirectional undulating curve, but this method has some limitations. First, graph consisted of a number of one-way data flow possess poor visual friendliness; Secondly, quadratic curve function need to specify a control point. If selected improperly, the overall effect will greatly affected. However, with the ever-changing data each year, the relative position of each element will change, as well as the thickness of the curve, resulting in difficulty in control point selection. The second solution can generate naturally undulating curve, however, the selection of the two control points increases the difficulty. Therefore, we need to find a drawing method getting rid of the constraints of specific values, which can simulate the Normative Cubic Curve.

\section{B. Basic Principles}

The paper focuses on the bending degrees and cross-cutting issues of data flow, and proposes a multi-arc seamless curve drawing method. The basic principle is: two pieces of quarter ellipse arcs tangent to each other simulate a segment of fairly standard cubic curve.

In the paper, each curve is divided into four segments: two pieces of straight lines at both ends, and two pieces of quarter ellipse arcs in the middle. The arcs are from two ellipses with same length and width and adjacent at their vertexes. Figure 1 shows the curve described above. 

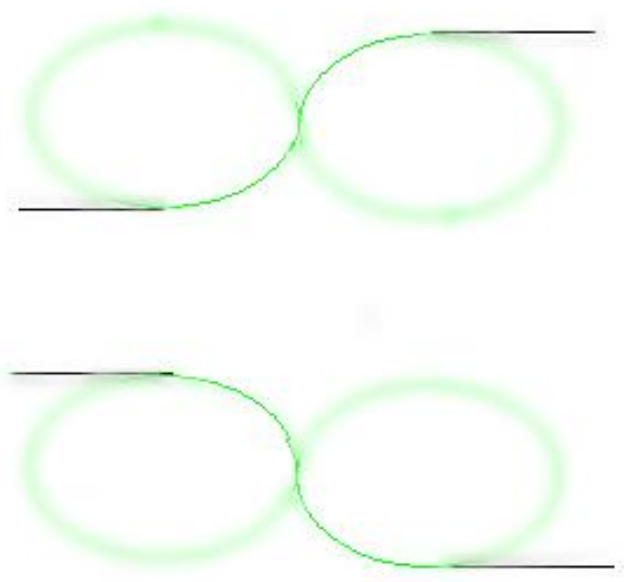

Figure 1. Curve drawing schematics

\section{Related Concepts and Algorithms}

1) Forward Curve Flow: The stream starting from the left side, imitating a river, flows from high to low. It is notated with PosFlow.

2) Reverse Curve Flow: The stream is defined in an against way to Forward Curve Flow, starting from the left side, flows from low to high.

Assume a rectangular region, which contains two identical half of ellipses. Let $\mathrm{p}(\mathrm{x} 0, \mathrm{y} 0)$ be the upper left vertex point of the rectangle, $\mathrm{p}(\mathrm{x} 1, \mathrm{y} 1)$ be the lower right vertex point, "arcWidth" and "arcHeight" be the major axis and minor axis of the ellipse respectively--"arc1Location" and "arc2Location" be where x-axis is tangent to each of the ellipses.

Thus:

- $\operatorname{arcWidth}=\mathrm{x} 1-\mathrm{x} 0$;

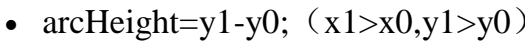

- $\operatorname{arc} 1$ Location $=(3 * x 0-x 1) / 2$;

- $\operatorname{arc} 2$ Location $=(x 0+x 1) / 2$;

For forward curve flows, the two arcs are:

- $\operatorname{arc}=$ new Arc2D.Float(arc1Location,y0,arcWidth, arcHeight,0,-90, Arc2D.OPEN);

- g2.draw (arc);

- $\operatorname{arc}=$ new Arc2D.Float(arc2Location,y0,arcWidth, arcHeight,180,-90, Arc2D.OPEN);

- g2.draw(arc);

For reverse curve flows, the two arcs are:

- $\operatorname{arc}=$ new Arc2D.Float(arc1Location,y0,arcWidth, arcHeight,0,90, Arc2D.OPEN);

- g2.draw(arc);

- $\operatorname{arc}=$ new Arc2D.Float(arc2Location,y0,arcWidth, arcHeight, 180,90, Arc2D.OPEN);

- g2.draw (arc);

\section{Validation}

The algorithm is based on the premise that: the width and high interval of rectangular frame be set in advance, which represents each element, namely alpha and beta; And height of rectangular frame be automatically calculated.

Thus, $\mathrm{p}(\mathrm{x} 0, \mathrm{y} 0)$ and $\mathrm{p}(\mathrm{x} 1, \mathrm{y} 1)$ are belong to controllable range, then the relevant variables, such as arcWidth, arcHeight, arc1Location, and arc2Location can get rid of specific numerical limits.

In addition, since any two rectangular region between elements from different groups will never overlap, so that the resulting curves are not possible to ensure overlap. Meanwhile, symmetry of curvature ensures the chart clear and precise.

\section{IV.SIMULATION}

According to the energy research framework, this paper draws a Sankey diagram based on energy flow data of year 2011. The data include primary energy input, power generation, and energy consumption, as shown in Table 1.

TABLE 1. ENERGY BALANCE TABLE OF 2011

\begin{tabular}{|l|l|l|l|l|}
\hline & Hydro & Nuclear & Wind & Coal \\
\hline Supply & 22166 & 2738 & 2981 & 252982 \\
\hline Imports & & & & 63426 \\
\hline $\begin{array}{l}\text { Generation } \\
\text { Investment }\end{array}$ & 22166 & 2738 & 2981 & 129971 \\
\hline $\begin{array}{l}\text { Loss \& } \\
\text { Statistical } \\
\text { Difference }\end{array}$ & & & & 16273 \\
\hline $\begin{array}{l}\text { Industry \& } \\
\text { Construction }\end{array}$ & & & & 94168 \\
\hline Transportation & & & & 458 \\
\hline Commercial & & & & 4670 \\
\hline Residential & & & & 7442 \\
\hline Net Exports & & & & \\
\hline
\end{tabular}

\begin{tabular}{|l|l|l|l|l|}
\hline & Petroleum & $\begin{array}{l}\text { Natural } \\
\text { Gas }\end{array}$ & $\begin{array}{l}\text { Power } \\
\text { Generate }\end{array}$ & $\begin{array}{l}\text { Thermal } \\
\text { Energy }\end{array}$ \\
\hline Supply & 65096 & 172810 & 162357 & 10361 \\
\hline Imports & 32101 & 39745 & & \\
\hline $\begin{array}{l}\text { Generation } \\
\text { Investment }\end{array}$ & 1135 & 3366 & & \\
\hline $\begin{array}{l}\text { Loss \& } \\
\text { Statistical } \\
\text { Difference }\end{array}$ & 2371 & 312 & 97052 & \\
\hline $\begin{array}{l}\text { Industry \& } \\
\text { Construction }\end{array}$ & 24997 & 7582 & 47840 & \\
\hline
\end{tabular}




\begin{tabular}{|l|l|l|l|l|}
\hline Transportation & 23233 & 16891 & 1111 & \\
\hline Commercial & 7120 & 816 & 6903 & \\
\hline Residential & 6240 & 3516 & 9296 & \\
\hline Net Exports & & & 157 & \\
\hline
\end{tabular}

The Energy Sankey diagram is shown in Figure 2.

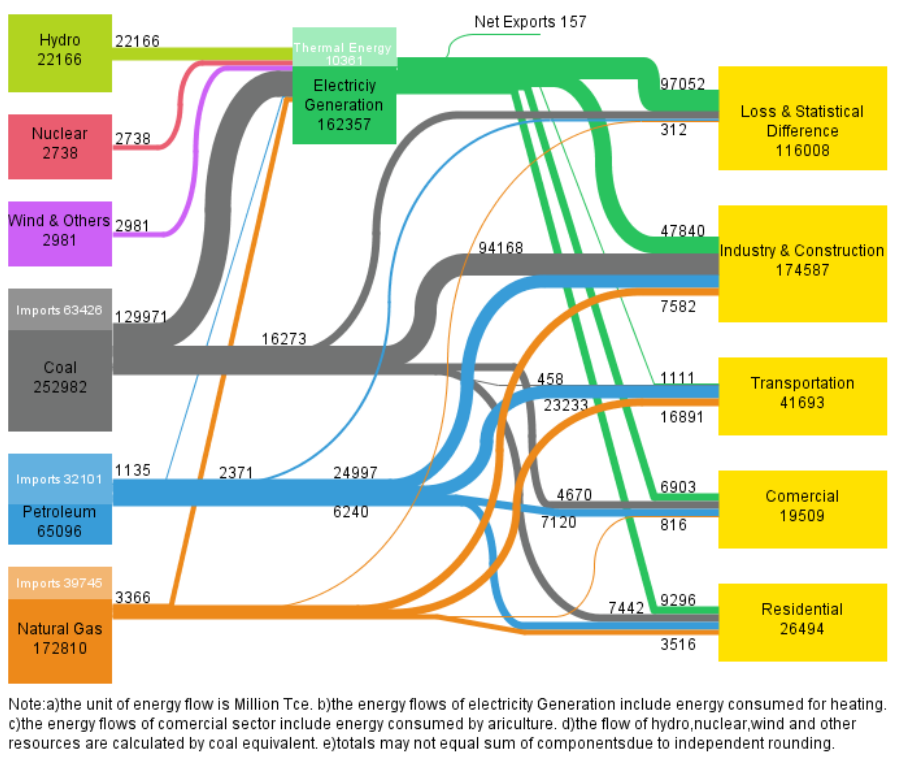

Figure 2. Energy Sankey diagram of year 2011

\section{Conclusions}

In the paper, a drawing method of Energy Sankey Diagram based on Java Graphics2D is approached. Meanwhile, a multi arc seamless bonding curve drawing method is introduced. Experiments show that the method can not only flexibly generate flow curves, but also reduce crosses between curves, which make the chart clear and precise.

With the era of big data, data analysis and visualization capabilities were taken seriously. Therefore, further functional requirements are raised to the energy Sankey diagram. Forecasting for data of the coming years, and drawing carbon flow diagram based on $\mathrm{CO}_{2}$ emission data is still a problem to be solved, as well as interactive features. Future research will focus on these issues.

\section{ACKNOWLEDGMENT}

Many people have made invaluable contributions, both directly and indirectly to this paper. Dr. Zhu offered help in energy research framework, who is really a patient tutor. Siqi He offered help in organizing functional requirements. Yue Zhao offered help in regulating the translation. It's really kind of her to do all this job. I would like to express my gratitude to all those colleagues in department of Big Data who have offered valuable suggestions in making the paper a reality.

\section{REFERENCES}

[1] S. Zhang, C. Zhu, J. K. O. Sin, and P. K. T. Mok, "A novel ultrathin elevated channel low-temperature poly-Si TFT," IEEE Electron Device Lett., vol. 20, pp. 569-571, Nov. 1999.

[2] Zheng.LI, Feng.Fu, Lin.wei. Ma, and Wei.dou. Ni, “ The China-based energy balance energy flow diagram,” Chinese Energy, vol. 09, pp.4-6, Nov.2006

[3] Hai.tao.Gong, Sheng.li.Ji, "The Sankey diagram in energy and material flow management," Journal of Industrial Ecology, vol. 12, pp. 173-185, Nov. 2008.

[4] McCalley. J, Krishnan.V, Gkritza.K, Brown.R, Mejia-Giraldo. D, "Planning for the Long Haul: Investment Strategies for National Energy and Transportation Infrastructures," Power and Energy Magazine, IEEE, vol.11, no.5, pp.24,35, Sept. 2013.

[5] Liu Wen-Chao,Wang Jian-jun,Cai Jiu-ju, "Analysis of Energy Flow and Its Modeling in Integrated Steelworks," Energy and Environment Technology, 2009. ICEET '09. International Conference on, vol.1, pp. 33,36, 16-18, Oct. 2009

[6] Ren Gui-zhou, Chang Si-qin, "Analysis and Research on Energy Flow Optimal Control and Allocation Strategy for Internal CombustionLinear Generator Integrated Power System," Electrical and Control Engineering (ICECE), 2010 International Conference on, vol., pp. 3316,3319, 25-27, June 2010

[7] Fouladgar, A.M, Simeone, O., "Information and Energy Flows in Graphical Networks with Energy Transfer and Reuse," Wireless Communications Letters, IEEE, vol.2, no.4, pp.371,374, August 2013.

[8] Pasic.J, "Fast detection of energy flow direction," MIPRO, 2011 Proceedings of the 34th International Convention, vol., pp.781,784, 23-27 May 2011.

[9] Riffonneau.Y, Bacha. S, Barruel. F, Delaille. A, "Energy flow management in grid connected PV systems with storage - A deterministic approach," Industrial Technology, 2009. ICIT 2009. IEEE International Conference on, vol., pp.1,6, 10-13 Feb. 2009./

[10] Hailun-Lin, Guiling-Wang, Peng-Zhang, Jing-Wang, Yanbo-Han, "A Two-Level Programming Model Based on Spreadsheet and Data Flow Chart," 2010 7th Web Information Systems and Applications Conference (WISA), vol., pp.39,42, 20-22 Aug. 2010.

[11] Li.G.-S, Tricoche. X, Weiskopf. D, Hansen. C, "Flow Charts Visualization of Vector Fields on Arbitrary Surfaces," Visualization and Computer Graphics, IEEE Transactions on , vol.14, pp.1067,1080, Sept.-Oct. 2008

[12] (2012) The esankey website. [Online]. Available: http://www.ifu.com/en/references/esankey/

[13] (2013) The sankey website. [Online]. Available: http://bost.ocks.org/mike/sankey/

[14] Yew.Kwang.Hooi, Leong.Siew.Yoong, "Technique to correct displacement of vector drawings on scaled underlying raster image," Computers \& Informatics (ISCI), 2011 IEEE Symposium on , vol., pp.102,107, 20-23 March 2011.

[15] (2011) The json website. [Online]. Available: http://www.json.org/js.html

[16] Dhawan. M, Ganapathy.V, "Analyzing Information Flow in JavaScript-Based Browser Extensions," Computer Security Applications Conference, 2009. ACSAC '09. Annual, vol., pp.382,391, 7-11 Dec. 2009

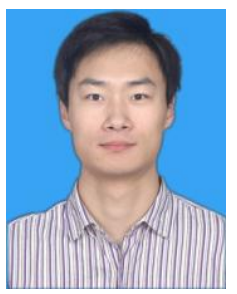

Chunyu Deng was born in Hebei China, 1982. He was graduated from North China Electric Power University, master, majoring in computer science and technology. Mr. Deng is devoted to the work of application of data mining in power industry. 


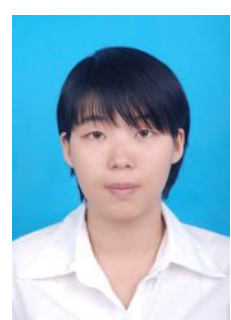

Hongmin Li was born in Hebei China, 1988. She was graduated from North China Electric Power University, master, majoring in computer science and technology. $\mathrm{Ms}$. $\mathrm{Li}$ is devoted to the work of data visualization in power industry.

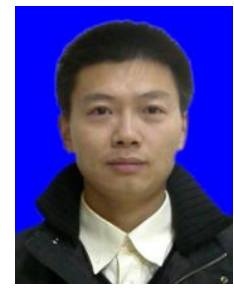

Yupeng Shao was born in Heilongjiang China, 1980. He was graduated from Shenyang Lu Xun Academy of Fine Arts, majoring in Art and Design Research Mr. Shao is devoted to the work of presentation Theory for big data visualization. 